M. Yamazato

Nagoya Math. J.

Vol. 119 (1990), 143-172

\title{
HITTING TIME DISTRIBUTIONS OF SINGLE POINTS FOR 1-DIMENSIONAL GENERALIZED DIFFUSION PROCESSES
}

\author{
MAKOTO YAMAZATO
}

\section{$\S 1$. Introduction}

In this paper, we will characterize the class of (conditional) hitting time distributions of single points of one dimensional generalized diffusion processes and give their tail behaviors in terms of speed measures of the generalized diffusion processes.

Kent [10] considered the characterization problem of the class of hitting time distributions and he got some partial results. Our Theorem 1 completely solves the characterization problem. Birth and death processes are regarded as a type of one dimensional generalized diffusions. The author [16] succeeded in characterizing the class of first passage time distributions of the processes. However, in [16], first passage times to (reachable) boundaries at infinity are not considered. In this paper we treat hitting times of single points including boundary points. So the result in this paper is an extension of [16] in this sense too.

Has'minskii [4] got a condition on speed measures of null recurrent diffusion processes for hitting time distributions of regular points to belong to domains of attraction of one dimensional one sided stable distributions and, under this condition, he obtained a limit theorem on occupation times of one dimensional null recurrent diffusion processes. The condition that he obtained is complicated. Theorem 4 of this paper simplifies his condition. However, the limit theorem on occupation time, which Has'minskii obtained, had earlier been obtained by Stone [14] under the same condition in Theorem 4, using another method.

Theorems 3-6 describe tail behaviors of hitting time distributions. In detail, Theorem 3 is a result on the case of exponential order decay and Theorems 4-6 are on the case of power order decay. Theorem 3(i) and Theorem 5 treat transient generalized diffusions, Theorem 4 treats

Received February 10, 1989. 
null recurrent ones and Theorem 3(ii) and Theorem 6 treat positive recurrent ones. All cases, except the case that masses of the speed measures near boundaries increase or decrease with integral power orders, are covered by Theorems 3-6.

In Section 2, we define one dimensional generalized diffusion processes and their (conditional) hitting time distributions of single points. Section 3 is devoted to the description of results on the characterization of the class of hitting time distributions and notions necessary to describe the results. Section 4 is devoted to the description of the tail behaviors of hitting time distributions. In Section 5, we summarize the theory of strings of Kac and Krein, which plays an essential role in proving our results. In Sections 6 and 7, we prove the results in Sections 3 and 4, respectively.

Acknowledgment. The author would like to thank Shin'ichi Kotani for useful discussion.

\section{§2. Generalized diffusion processes}

Let $\{B(t)\}$ be a one-dimensional Brownian motion and let $l(t, x)$ be its local time. We denote by $\mathscr{M}$ the class of right continuous nondecreasing function $m$ on $[-\infty, \infty]$ to $[-\infty, \infty]$ with $m(-\infty)=-\infty, m(+\infty)=+\infty$ and $m(0-)=0$. For $m \in \mathscr{M}$, we define $l_{1}=l_{1}(m)$ and $l_{2}=l_{2}(m)$ by

$$
(-1)^{i} l_{i}=\inf \left\{(-1)^{i} x>0 ;(-1)^{i} m(x)=\infty\right\}
$$

for $i=1,2$, and we define a measure $m(d x)$ on $[-\infty, \infty]$ by

$$
\begin{aligned}
& m(d x)=d m(x) \quad \text { on }\left(l_{1}, l_{2}\right), \\
& m\left(\left[l_{1}, l_{2}\right]^{c}\right)=0
\end{aligned}
$$

and

$$
m\left(\left\{l_{t}\right\}\right)=\infty \quad \text { for } \quad i=1,2 \text {. }
$$

Here, $\left[l_{1}, l_{2}\right]^{c}$ is the complement of $\left[l_{1}, l_{2}\right]$. Let

$$
\phi(t)=\int_{R} l(t, x) m(d x) .
$$

Define a stochastic process $\{X(t), \zeta\}$ by $X(t)=B\left(\phi^{-1}(t)\right)$ and the life time

$$
\begin{aligned}
\zeta & =\inf \left\{t>0 ; X(t)=l_{1} \text { or } l_{2}\right\} \text { in the case }\{\} \neq \phi, \\
& =\infty \text { otherwise. }
\end{aligned}
$$

This process is strong Markov with state space $E_{m}=\left.(\operatorname{supp} m)\right|_{\left(l_{1}, l_{2}\right)}$ and is 
called the generalized diffusion process corresponding to the function $m$ (see [12] or [5]). In this paper, we abbreviate generalized diffusion process as g.d.p. The measure $m$ restricted to $\left(l_{1}, l_{2}\right)$ is called the speed measure of the process $\{X(t)\}$. For $y \in E_{m}$, we define the hitting time of $y$ by

$$
\tau_{v}=\inf \{t>0 ; X(t)=y\}
$$

if there is $t>0$ such that $X(t)=y$ and set $\tau_{y}=\infty$ if otherwise. If $\left|l_{i}\right|<\infty$ and $l_{i} \in \bar{E}_{m}$, where $\bar{E}_{m}$ is the closure of $E_{m}$ in $\mathbf{R}$, then we define $\tau_{l_{i}}$ by $\lim _{y \rightarrow l_{i}} \tau_{y}$ for $i=1,2$. We denote by $\tilde{E}_{m}$ the set with $l_{i}(i=1,2)$ adjoined to $E_{m}$ whenever $\left|l_{i}\right|<\infty$ and $l_{i} \in \bar{E}_{m}$. If $P_{x}\left(\tau_{y}<\infty\right)>0$ for $x$ in $E_{m}$ and $y$ in $\tilde{E}_{m}$, we say that $\mu_{x y}(d t)=P_{x}\left(\tau_{y} \in d t\right) / P_{x}\left(\tau_{y}<\infty\right)$ is the conditional hitting time distribution of $y$ starting at $x$. We always assume that $x \neq y$ when we discuss conditional hitting time distributions. We denote by

$$
H_{g d}=\left\{\mu_{x y}(d y) ; x \in E_{m}, y \in \tilde{E}_{m}, x \neq y, m \in \mathscr{M}\right\},
$$

the class of conditional hitting time distributions of generalized diffusion processes.

\section{§3. Characterization of $H_{g d}$}

Let $\mathbf{R}_{+}=[0, \infty)$ and let $\mathscr{P}\left(\mathbf{R}_{+}\right)$be the class of probability distributions on $\mathbf{R}_{+}$. We denote the Laplace transform of $\mu \in \mathscr{P}\left(\mathbf{R}_{+}\right)$by $\mathscr{L} \mu(\lambda)=\int e^{-\lambda x} \mu(d x)$. Let $C E_{+}$be the smallest subclass of $\mathscr{P}\left(\mathbf{R}_{+}\right)$, which contains all exponential distributions and closed under convolution and weak convergence. A probability measure $\mu$ on $\mathbf{R}_{+}$is a $C E_{+}$distribution if $\mu$ is concentrated at a point in $\mathbf{R}_{+}$, or there are $\gamma \geqq 0$ and a nondecreasing sequence $\left\{a_{t}\right\}$ of at most countable positive numbers satisfying $\sum_{i} a_{i}^{-1}$ such that

$$
\mathscr{L} \mu(\lambda)=e^{-r \lambda} \prod_{i} a_{i}\left(\lambda+a_{i}\right)^{-1}
$$

We call $\left\{a_{i}\right\}$ the parameter sequence of $\mu$. We denote by $C E_{+0}$ the class of distributions $\mu$ in $C E_{+}$whose Laplace transform is represented as (3.1) with $\gamma=0$. We say that a probability measure $\mu$ is a $M E_{+}$distribution if there is a probability measure $G$ on $(0, \infty]$ such that

$$
\mu([0, x])=\int_{(0, \infty]}\left(1-e^{-x u}\right) G(d u) \quad \text { for } \quad x>0 .
$$

We call $G$ the mixing distribution of $\mu$. We denote by $M E_{+0}$ the class of 
distributions $\mu$ in $M E_{+}$such that $\mu(\{0\})=0$. For $\mu \in M E_{+}$, the condition $\mu(\{0\})=0$ is equivalent to $G(\{\infty\})=0$ for the mixing measure $G$ of $\mu$. Let $C M E_{+00}\left(C M E_{+}\right.$resp.) be the class of distributions $\mu=\mu_{1} * \mu_{2}$ with $\mu_{1} \in C E_{+0}\left(C E_{+}\right.$resp.) and $\mu_{2} \in M E_{+0}\left(M E_{+}\right.$resp.). Here $*$ denotes convolution. Let $\boldsymbol{B O}$ be the smallest class of distributions on $\mathbf{R}_{+}$which contains $M E_{+}$ and closed under convolution and weak convergence. It is known that a probability measure $\mu$ on $\mathbf{R}_{+}$belongs to the class $\boldsymbol{B O}$ if and only if there are $\gamma \geqq 0$ and a measure $Q$ on $(0, \infty)$ satisfying $\int_{(0, \infty)} \frac{1}{u(u+1)} Q(d u)$ $<\infty$ such that

$$
\mathscr{L} \mu(\lambda)=\exp \left\{-\gamma \lambda+\int_{(0, \infty)}\left(\frac{1}{u+\lambda}-\frac{1}{u}\right) Q(d u)\right\}
$$

([1]). We call the measure $Q Q$-measure of $\mu \in \boldsymbol{B O}$. The $Q$-measure of a gamma distribution with density

$$
\begin{aligned}
& f(x)=\Gamma(\alpha)^{-1} x^{\alpha-1} e^{-x} \quad \text { for } \quad x>0, \\
& =0 \quad \text { for } x<0 \text {, }
\end{aligned}
$$

where $\alpha>0$, is absolutely continuous and the density $q$ is given by the following:

$$
\begin{aligned}
q(u)=0 & \text { if } & 0<u<a, \\
=\alpha & \text { if } & u>a .
\end{aligned}
$$

The representation (3.2) for $\mu \in M E_{+}$is characterized in the following. A distribution $\mu$ on $\mathbf{R}_{+}$belongs to $M E_{+}$if and only if, in the representation (3.2) of the Laplace transform of $\mu, \gamma=0, Q$ satisfies $\int_{(0,1)} \frac{1}{u} Q(d u)<\infty$, $Q$ is absolutely continuous and its density is bounded by 1 a.e. By the definitions of the classes $C M E_{+}$and $\boldsymbol{B O}$, it is clear that $C M E_{+} \subset \boldsymbol{B O}$. Kent [10] showed that $H_{g d} \subsetneq B \boldsymbol{B O}$. On the other hand, Keilson [9] showed that the first passage time distributions of birth and death processes are contained in $C M E_{+}$. Moreover, he showed that the number of exponential distributions arising in the convolution is finite. The author [16] refined Keilson's result and determined the class of first passage time distributions of birth and death processes. The author also remarked that $H_{g d} \subset C M E_{+}$ by approximating g.d.p. by birth and death processes.

We denote by $\mathbf{C}$ the plane of complex numbers and denote by $\mathbf{C}_{+}\left(\mathbf{C}_{-}\right)$ the open upper (lower) half plane in $\mathbf{C}$. Let $\mathscr{H}_{0}$ be the class of nonnegative valued functions $h(\lambda)$ on $(0, \infty)$ which is extendable to holomorphic functions 
$\tilde{h}(\lambda)$ on $\mathbf{C} \backslash(-\infty, 0]$ such that $\tilde{h}\left(\mathbf{C}_{+}\right) \subset \mathbf{C}_{-}$. Let $\mathscr{H}=\mathscr{H}_{0} \cup\{h \equiv \infty\}$. The following representation is well known.

LEMma 3.1. In order that a function $h$ on $(0, \infty)$ belongs to $\mathscr{H}_{0}$, it is necessary and sufficient that there are $c \geqq 0$ and a nonnegative measure $\sigma$ on $[0, \infty)$ satisfying $\int_{[0, \infty)} \frac{\sigma(d \xi)}{1+\xi}<\infty$ such that

$$
h(\lambda)=c+\int_{[0, \infty)} \frac{\sigma(d \xi)}{\lambda+\xi} .
$$

The measure $\sigma$ is called the spectral measure of $h$.

Remark 3.1. If $\mu \in M E_{+}$, then $\mathscr{L} \mu(\lambda)$ belongs to $\mathscr{H}_{0}$ and is represented as (3.4) with $c=G(\{\infty\})$ and $\sigma(d \xi)=\xi G(d \xi)$ on $(0, \infty)$ by the mixing distribution $G$ of $\mu$. Conversely, $h \in \mathscr{H}_{0}$ with $h(0)=1$ is the Laplace transform of an $M E_{+}$distribution.

Remark 3.2 ([2] p. 27). It is known that $h \in \mathscr{H}_{0}$ if and only if there are a real number $\alpha$ and a measurable function $q(u)$ satisfying $0 \leqq q(u) \leqq 1$ such that

$$
h(\lambda)=\exp \left\{\alpha+\int_{(0, \infty)}\left(\frac{1}{u+\lambda}-\frac{u}{u^{2}+1}\right) q(u) d u\right\}
$$

for $\lambda>0$. We also call the measure $q(u) d u Q$-measure of $h \in \mathscr{H}_{0}$.

Lemma 3.2. If $h \in \mathscr{H}_{0}$ and $h \neq 0$, then $(\lambda h(\lambda))^{-1} \in \mathscr{H}_{0}$. The $Q$-measure $\tilde{Q}$ of $(\lambda h(\lambda))^{-1}$ is represented as $(1-q(x)) d x$ by the Q-measure $q(x) d x$ of $h$.

Proof. It is easy to check that if $h \in \mathscr{H}_{0}$ and $h \neq 0$, then $(\lambda h(\lambda))^{-1} \in \mathscr{H}_{0}$. We easily confirm that

$$
\frac{1}{\lambda}=\exp \left\{\int_{(0, \infty)}\left(\frac{1}{u+\lambda}-\frac{u}{u^{2}+1}\right) d u\right\} \quad \text { for } \quad \lambda>0 .
$$

By Remark 3.2, we obtain

$$
(\lambda h(\lambda))^{-1}=\exp \left\{\alpha+\int_{(0, \infty)}\left(\frac{1}{u+\lambda}-\frac{u}{u^{2}+1}\right)[1-q(u)] d u\right\} .
$$

Now we can describe our main result on characterization problem.

Theorem 1. In order that a probability measure $\mu$ on $\mathbf{R}_{+}$belongs to $H_{g d}$, it is necessary and sufficient that there are a $C E_{+0}$ distribution $\mu_{1}$ and a $M E_{+0}$ distribution $\mu_{2}$ such that 


$$
\mu=\mu_{1} * \mu_{2},
$$

and the parameter sequence $\left\{a_{i}\right\}$ in the representation (3.1) of $\mathscr{L} \mu_{1}(\lambda)$ is either empty or strictly increasing and the spectral measure $\sigma$ of $\left(\lambda \mathscr{L} \mu_{2}(\lambda)\right)^{-1}$ has a positive point mass at $a_{i}$ for each $i$.

Corollary 1. Let $\mu \in M E_{+}$. Then $\mu \in H_{g d}$ if and only if $\mu(\{0\})=0$.

This corollary is immediate from Theorem 1. The "only if" part is also immediate from the fact that $P_{x}\{X(0+)=y\}=0$ for $x \neq y$.

Corollary 2. Let $\mu$ be a gamma distribution with exponent $\alpha>0$, i.e., $\mathscr{L} \mu(\lambda)=(a /(\lambda+a))^{\alpha}$ where $a>0$. Then, $a>0$. Then, $\mu \in H_{g d}$ if and only if $\alpha \leqq 1$.

Combining Theorem 1 with Corollary 1 or 2, we get the following.

Corollary 3. $H_{g d} \subsetneq C M E_{+00}$.

The condition in Theorem 1 is not easy to check. If we can prove the following conjecture, then we can give another necessary and sufficient condition which is easier to check.

Conjecture. Let $\mu \in M E_{+}$and let

$$
\mathscr{L} \mu(\lambda)=\int_{(0, \infty]} \frac{\xi}{\lambda+\xi} G(d \xi) .
$$

The mixing measure $G$ has a point mass at $\alpha<\infty$ if and only if $Q$-measure $Q$ of $\mu$ satisfies $Q[a-\varepsilon, a]=o(\varepsilon)$ and $Q[a, a+\varepsilon] \sim \varepsilon$ as $\varepsilon \downarrow 0$.

If the conjecture is true, then we have the following characterization of $H_{g d}$ : In order that $\mu \in \mathscr{P}\left(R_{+}\right)$belongs to $H_{g d}$ it is necessary and sufficient that there are $\mu_{1} \in C E_{+0}$ and $\mu_{2} \in M E_{+0}$ such that $\mu=\mu_{1} * \mu_{2}$, the parameter sequence $\left\{a_{i}\right\}$ of $\mu_{1}$ is either empty or strictly increasing and the Q-measure $Q$ of $\mu_{0}$ satisfies $Q[a-\varepsilon, a] \sim \varepsilon$ and $Q[a, a+\varepsilon]=o(\varepsilon)$ as $\varepsilon \downarrow 0$ for each $a \in\left\{a_{i}\right\}$.

This can be shown as follows. Since $Q$-measure $\tilde{Q}$ of $\left(\lambda \mathscr{L} \mu_{2}(\lambda)\right)^{-1}$ is given by $\tilde{Q}(d x)=d x-Q(d x)$ (see Lemma 3.2), the condition that $Q[a-\varepsilon, a]$ $\sim \varepsilon$ and $Q[a, a+\varepsilon]=o(\varepsilon)$ as $\varepsilon \downarrow 0$ is equivalent to the condition that $\tilde{Q}[a-\varepsilon, a]=o(\varepsilon)$ and $\tilde{Q}[a, a+\varepsilon] \sim \varepsilon$ as $\varepsilon \downarrow 0$. If the conjecture is true, the last condition is equivalent to that the spectral measure of $\left(\lambda \mathscr{L} \mu_{2}(\lambda)\right)^{-1}$ has a point mass at $a$. 
We say that a measure on $\mathbf{R}_{+}$is discrete if its support is either a finite set or a countable set having no accumulation point in $\mathbf{R}_{+}$. The conjecture is true in the following cases.

Case 1. The mixing measure $G$ is discrete.

Case 2. The mixing measure $G$ is written as $G=G_{1}+G_{2}$, where $G_{1}$ is discrete and $G_{2}$ is absolutely continuous with a density of $\mathrm{C}^{1}$-class.

We say that $\mu \in \mathscr{P}\left(\mathbf{R}_{+}\right)$is a $C M E_{+00}^{d}$ distribution (the superscript $d$ stands for discrete) if there are $\mu_{1} \in C E_{+0}$ and $\mu_{2} \in M E_{+0}$ such that $\mu=\mu_{1} * \mu_{2}$, the parameter sequence $\left\{a_{k}\right\}$ of $\mu_{1}$ is empty or strictly increasing, the mixing distribution $G$ of $\mu_{2}$ is discrete and (supp $\left.G\right) \cap\left\{a_{k}\right\}=\phi$. The Laplace transform $\mathscr{L} \mu(\lambda)$ is represented as follows:

$$
\mathscr{L} \mu(s)=\prod_{k} a_{k}\left(s+a_{k}\right)^{-1} \prod_{k} c_{k}^{-1}\left(s+c_{k}\right) b_{k}\left(s+b_{k}\right)^{-1}
$$

for finite or infinite strictly increasing sequences $\left\{a_{k}\right\},\left\{b_{k}\right\}$ and $\left\{c_{k}\right\} \quad\left(\left\{a_{k}\right\}\right.$ may be empty) satisfying $\sum a_{k}^{-1}<\infty$ in the case $\left\{a_{k}\right\} \neq \phi,\left\{a_{k}\right\} \cap\left\{b_{k}\right\}=\phi$, $b_{k}<c_{k}<b_{k+1}$ for each $k$,

$$
1 \leqq \sharp\left\{c_{k}\right\}=\sharp\left\{b_{k}\right\}-1 \quad \text { in the case } \sharp\left\{b_{k}\right\}<\infty
$$

and

$$
\prod_{k} \frac{b_{k}}{c_{k}} \text { diverges to } 0 \quad \text { in the case } \sharp\left\{b_{k}\right\}=\infty \text {. }
$$

Here $\#\{\}$ stands for the cardinality of a set \{\} .

Remark 3.3. The above representation (3.5) of $\mathscr{L} \mu(\lambda)$ is not unique. We call $\left(\left\{a_{k}\right\},\left\{b_{k}\right\},\left\{c_{k}\right\}\right)$ a minimal representation of $\mu$ if $\left\{a_{i}\right\} \cap\left\{c_{i}\right\}=\phi$, since in this case $\left\{a_{i}\right\}$ is minimal in a component wise sense (see [16]).

Theorem 2. (i) Assume that $m \in \mathscr{M}, x \in E_{m}$ and $y \in \tilde{E}_{m}$. If one of the following three conditions is satisfied, then the conditional hitting time distribution $\mu_{x y}$ of the g.d.p. corresponding to $m$ belongs to $C M E_{+00}^{d}$.

1. $l_{1}>-\infty$ and $m\left(l_{1}+\right)>-\infty$.

2. $l_{1}>-\infty, m\left(l_{1}+\right)=-\infty$ and $\lim _{u \rightarrow l_{1}}\left(u-l_{1}\right) m(u)=0$.

3. $l_{1}=-\infty, m((-\infty)+)>-\infty$ and $\lim _{u \rightarrow-\infty} u\{m(u)-m((-\infty)+)\}=0$.

(ii) For $\mu \in C M E_{+00}^{d}$, there is $m \in \mathscr{M}$ satisfying one of the conditions 1,2 and 3 (i) such that $0 \in E_{m}, 1 \in \tilde{E}_{m}$ and $\mu$ is the conditional hitting time distribution of 1 starting at 0 of the g.d.p. corresponding to $m$. 


\section{§4. Tail behaviors}

Lemma 4.1. Let $m \in \mathscr{M}, 0 \in E_{m}$ and $b \in \tilde{E}_{m}$. Then, $P_{0}\left(\tau_{b}<\infty\right)>0$ for the g.d.p. corresponding to $m$ if and only if $\int_{0}^{b-} m(x) d x<\infty$.

The proof of this lemma will be given in Section 6 . In this section, we assume that $m \in \mathscr{M}, 0 \in E_{m}$ and $\mu$ is the conditional hitting time distribution of $b \in \tilde{E}_{m}, b>0$, of the g.d.p. corresponding to $m$, starting at 0 . Since $\int_{0}^{b-} m(x) d x<\infty$ and $(b-x) m(x) \leqq \int_{x}^{b-} m(y) d y,(b-x) m(x)$ is bounded in $x \in[0, b)$.

Theorem 3. Let $A=\sup _{x \in[0, b)}(b-x) m(x)$. (i) If $l_{1}>-\infty$ and there is $B>0$ such that

$$
\left|\left(x-l_{1}\right) m(x)\right| \leqq B \quad \text { for all } x \in\left(l_{1}, 0\right),
$$

then for every $\alpha$ satisfying $0<\alpha<\frac{1}{4} \cdot \min \{1 / A, 1 / B\}$,

$$
\mu([t, \infty))=o\left(e^{-\alpha t}\right) \quad \text { as } t \rightarrow \infty .
$$

(ii) If $l_{1}=-\infty, m((-\infty)+)>-\infty$ and there is $C>0$ such that

$$
|x\{m(x)-m((-\infty)-)\}| \leqq C \quad \text { for all } x \in(-\infty, 0]
$$

then there is $\beta$ in $(0, \min \{1 / A, 1 / C\})$ and $M>0$ such that

$$
\mu([t, \infty)) \sim M e^{-\beta t} \quad \text { as } t \rightarrow \infty .
$$

TheOREM 4. Let $0<\alpha<1$. Let $K(x)$ be a positive function slowly varying at infinity. If $l_{1}=-\infty$ and

$$
|m(-x)| \sim x^{\alpha-1-1} K(x) \quad \text { as } x \rightarrow \infty,
$$

then

$$
\mu([t, \infty)) \sim b\{\alpha(1-\alpha)\}^{\alpha} t^{-\alpha} / \Gamma(1+\alpha) L_{\alpha}(t) \quad \text { as } t \rightarrow \infty,
$$

where $t^{\alpha} L_{\alpha}(t)$ is an asymptotic inverse function ([13]) of $x^{\alpha-1} K(x)$.

THEOREM 5. If $l_{1}>-\infty$ and if, for a non-integral $\alpha>0$.

$$
|m(-x)| \sim\left(\left|l_{1}\right|-x\right)^{-\alpha-1-1} K\left(\left|l_{1}\right|-x\right) \quad \text { as } x \uparrow\left|l_{1}\right|,
$$

where $K(x)$ is a function slowly varying at 0 , then

$$
\mu([t, \infty)) \sim t^{-a} L(t) \quad \text { as } t \rightarrow \infty,
$$

with a function $L(t)$ slowly varying at infinity. 
Theorem 6. If $l_{1}=-\infty, m((-\infty)+)>-\infty$ and if, for a non-integral $\alpha>0$,

$$
|m((-\infty)+)-m(-x)| \sim x^{(\alpha+1)-1-1} K(x) \quad \text { as } x \rightarrow \infty,
$$

where $K(x)$ is a function slowly varying at infinity, then

$$
\mu([t, \infty)) \sim t^{-\alpha-1} L(t) \quad \text { as } t \rightarrow \infty,
$$

with a function $L(t)$ slowly varying at infinity.

\section{§5. Krein's correspondence and generalized diffusion processes}

Let $\mathscr{M}_{+}$be the class of $[0, \infty]$-valued nondecreasing right continuous functions on $[0, \infty]$ satisfying $m(\infty)=\infty$. Let $l(m)=\min \{x \geqq 0 ; m(x)=\infty\}$. Let $\varphi(x, \lambda)$ and $\psi(x, \lambda)$ be the continuous solutions of

$$
\varphi(x, \lambda)=1+\lambda \int_{[0, x)}(x-y) \varphi(y, \lambda) d m(y) \quad \text { for } x>0
$$

and

$$
\psi(x, \lambda)=x+\lambda \int_{[0, x)}(x-y) \psi(y, \lambda) d m(y) \quad \text { for } x>0
$$

respectively. Here $m(\{0\})$ is defined by $m(0)$. Since

$$
\frac{d^{+}}{d x}\left(\frac{\psi(x, \lambda)}{\varphi(x, \lambda)}\right)=\frac{1}{\varphi(x, \lambda)^{2}},
$$

$\lim _{x \rightarrow l} \psi r(x, \lambda) / \varphi(x, \lambda)$ exists and we denote this limit by $h(\lambda)$. Here we denoted right derivative by $d^{+} / d x$. It is known that $h \in \mathscr{H}$. Define a map on $\mathscr{M}_{+}$to $\mathscr{H}$ by the above correspondence and denote it by $\Phi$. This is called Krein's correspondence. It is known that this map is onto and one-to-one ([3], [6]).

Remark 5.1. $h \equiv \infty$ and $h \equiv 0$ correspond to $m \equiv 0$ and $m \equiv \infty$, respectively.

Remark 5.2. Let $m \in \mathscr{M}_{+}$and $h=\Phi(m)$. Let $\sigma$ be the spectral measure of $h$ and let $c$ be the number appearing in the representation (3.4) of $h$. Then $c=h(\infty)=\inf \{x \geqq 0 ; m(x)>0\}$ and

$$
l(m)=c+\int \frac{\sigma(d \xi)}{\xi}=h(0+) .
$$

Lemma 5.1 ([7]). If $h \in \mathscr{H}$ corresponds to $m \in \mathscr{M}_{+}$, then $(\lambda h(\lambda))^{-1}=$ $\Phi\left(m^{-1}\right)(\lambda)$ where $m^{-1}(x)=\inf \{y>0 ; m(y)>x\} \in \mathscr{M}_{+}$. 
For $m \in \mathscr{M}_{+}$, let $\sigma$ be the spectral measure of $\Phi(m)$. Set $\lambda_{0}(m)=$ $\inf (\operatorname{supp} \sigma)$. If $\lambda_{0}(m)=0$, then we define $\lambda_{1}(m)$ by $\lambda_{1}(m)=\inf \left\{\left.(\operatorname{supp} \sigma)\right|_{(0, \infty)}\right\}$. Let

$$
\begin{aligned}
C(m) & =\sup _{0 \leqq x<l(m)}(l(m)-x) m(x) & & \text { if } l(m)<\infty, \\
& =\infty & & \text { if } l(m)=\infty .
\end{aligned}
$$

In the case $l(m)=\infty$, let

$$
\begin{aligned}
D(m) & =\sup _{0 \leqq x<\infty} x(m(\infty-)-m(x)) & & \text { if } m(\infty-)<\infty, \\
& =\infty & & \text { if } m(\infty-)=\infty .
\end{aligned}
$$

Lemma 5.2 ([7]). Let $m \in \mathscr{M}$. Then

$$
C(m) \leqq \lambda_{0}(m)^{-1} \leqq 4 C(m) .
$$

LEMma 5.3 ([7]). Let $m \in \mathscr{M}_{+}$and let $\sigma$ be the spectral measure of $\Phi(m)$. Then the following (i) and (ii) hold.

$$
\begin{aligned}
\sigma(\{0\}) & =\lim _{\lambda \downarrow 0} \lambda h(\lambda)=1 / m(\infty-) & & \text { if } l(m)=\infty, \\
& =0 & & \text { if } l(m)<\infty .
\end{aligned}
$$

(ii) If $l(m)=\infty$ and $m(\infty-)<\infty$, then

$$
D(m) \leqq \lambda_{1}(m)^{-1} \leqq 4 D(m) .
$$

Lemma 5.4 ([11]). Suppose that $m \in \mathscr{M}_{+}$such that $l(m)=\infty$ and $m(\infty-)<\infty$. Put $h=\Phi(m)$ and let

$$
h^{*}(\lambda)=h(\lambda)-\sigma(\{0\}) / \lambda
$$

where $\sigma$ is the spectral measure of $h$. Then $m^{*}=\Phi^{-1}\left(h^{*}\right)$ and $l^{*}=l\left(m^{*}\right)$ are of the form

$$
m^{*}(x)=\frac{m(\infty-) m(t(x))}{m(\infty-)-m(t(x))}
$$

and

$$
l^{*}=\int_{0}^{\infty} \frac{\{m(\infty-)-m(s)\}^{2}}{m(\infty)^{2}} d s
$$

where $t(x)$ is the inverse function of

$$
x(t)=\int_{0}^{t} \frac{\{m(\infty-)-m(s)\}^{2}}{m(\infty)^{2}} d s .
$$


Lemma 5.5 ([7]). Let $m \in \mathscr{M}_{+}$. The spectral measure $\sigma$ of $\Phi(m)$ is discrete if and only if one of the following holds.

1. $l=l(m)<\infty$ and $m(l-)<\infty$.

2. $l<\infty, m(l-)=\infty$ and $\lim _{x \rightarrow l}(l-x) m(x)=0$.

3. $l=\infty, m(\infty-)<\infty$ and $\lim _{x \rightarrow \infty} x(m(\infty-)-m(x))=0$.

Lemma 5.6 ([7]). Let $m \in \mathscr{M}_{+}$. The spectral measure $\sigma$ of $\Phi(m)$ is discrete and its support $\left\{\lambda_{n}\right\}$ satisfies $\sum 1 / \lambda_{n}<\infty$ if and only if one of the following holds:

1. $l=l(m)<\infty$ and $m(l-)<\infty$.

2. $l<\infty, m(l-)=\infty$ and $\int_{0}^{l-} m(x) d x<\infty$.

3. $l=\infty$ and $\int_{0}^{\infty} x d m(x)<\infty$.

Lemma 5.7 ([10]). Let $m \in \mathscr{M}_{+}$. If $0<x<\infty$ and $\int^{x-} m(x) d x<\infty$, then the solutions $\varphi(x, \lambda)$ and $\psi(x, \lambda)$ if (5.1) and (5.2) are constant functions or entire functions of $\lambda$ such that their zero points $\left\{-a_{i}\right\}$ and $\left\{-b_{i}\right\}$ are negative, simple $\left(a_{i} \neq a_{j}\right.$ and $b_{i} \neq b_{j}$ if $\left.i \neq j\right)$ and satisfy $\sum a_{i}^{-1}<\infty$ and $\sum b_{\imath}^{-1}<\infty$. Moreover, $\varphi$ and $\psi$ are represented as

$$
\varphi(x, \lambda)=\prod\left(1+\frac{\lambda}{a_{i}}\right)
$$

and

$$
\psi(x, \lambda)=x \Pi\left(1+\frac{\lambda}{b_{i}}\right)
$$

Lemma 5.8 ([8]). If, for $m \in \mathscr{M}_{+}$,

$$
m(x) \sim x^{\alpha-1-1} K(x) \quad \text { as } x \rightarrow \infty,
$$

where $0 \leq \alpha<1$ and $K(x)$ is a function slowly varying at $\infty$, then

$$
\Phi(m)(\lambda) \sim D_{\alpha} \lambda^{-\alpha} L_{\alpha}\left(\lambda^{-1}\right) \quad \text { as } \lambda \rightarrow 0
$$

where $D_{\alpha}=\{\alpha(1-\alpha)\}^{-\alpha} \Gamma(1+\alpha) \Gamma(1-\alpha)^{-1}$ and $x^{\alpha} L_{\alpha}(x)$ is the asymptotic inverse at $\infty$ of $x^{\alpha-1} K(x)$.

All these facts except Lemmas 5.5-7 are found in Kotani-Watanabe $[12]$. 


\section{§. The proofs of Theorems 1 and 2}

Let $m \in \mathscr{M}$ and let $\{X(t), \zeta\}$ be a g.d.p. on $E_{m}$ corresponding to $m$. Let $b>0$ belong to $\tilde{E}_{m}$. Let $\varphi(x, \lambda)$ and $\psi(x, \lambda)$ be the continuous solutions of

$$
\begin{aligned}
\varphi(x, \lambda) & =1+\lambda \int_{[0, x)}(x-y) \varphi(y, \lambda) d m(y) & & \text { for } x>0 \\
& =1-\lambda \int_{[x, 0)}(x-y) \varphi(y, \lambda) d m(y) & & \text { for } x<0
\end{aligned}
$$

and

$$
\begin{aligned}
\psi(x, \lambda) & =x+\lambda \int_{[0, x)}(x-y) \psi(y, \lambda) d m(y) & & \text { for } x>0 \\
& =x-\lambda \int_{[x, 0)}(x-y) \psi(y, \lambda) d m(y) & & \text { for } x<0,
\end{aligned}
$$

respectively. Let

$$
\begin{aligned}
& h_{1}(\lambda)=-\lim _{x \rightarrow l_{1}} \psi(x, \lambda) / \varphi(x, \lambda) \quad \text { and } \\
& \tilde{h}_{2}(\lambda)=\lim _{x \rightarrow l_{2}} \psi(x, \lambda) / \varphi(x, \lambda) .
\end{aligned}
$$

Moreover, let

$$
m_{1}(x)=-m((-x)-)
$$

and

$$
m_{2}(x)=m(x) \quad \text { for } x>0 .
$$

Then $h_{1}(\lambda)=\Phi\left(m_{1}\right)$ and $\tilde{h}_{2}(\lambda)=\Phi\left(m_{2}\right)$. Define $u(x, \lambda)$ by

$$
u(x, \lambda)=\varphi(x, \lambda)+h_{1}(\lambda)^{-1} \psi(x, \lambda) .
$$

Then, for $\lambda>0, u$ is a positive increasing function of $x$, satisfying either $u\left(l_{1}+, \lambda\right)=0$ or $\left(d^{+} / d x\right) u((-\infty)+, \lambda)=0$ according as $l_{1}=l_{1}(m)>-\infty$ or $l_{1}=-\infty$. So, by the general theory on diffusion processes, the Laplace transform of $\tau_{b}$ is given by the following:

$$
\begin{aligned}
E_{0}\left(e^{-\lambda \tau b}\right) & =u(0, \lambda) / u(b, \lambda)=u(b, \lambda)^{-1} & & \text { if } b<l_{2}=l_{2}(m) \text { and } \\
& =\lim _{x \rightarrow \delta} u(x, \lambda)^{-1} & & \text { if } b=l_{2}<\infty .
\end{aligned}
$$

See Ito-McKean [4] p. 128-129.

Proof of Lemma 4.1. Note that since $\varphi(x, \lambda) \geqq 1$ for $\lambda>0$,

$$
\varphi(x, \lambda) \geqq 1+\lambda \int_{[0, x)} m(y) d y \quad \text { for } \lambda>0 .
$$


If $b=l_{2}(m)<\infty$ and $\int^{b-} m(x) d x=\infty$, then $\varphi(b-, \lambda)=\infty$. This means that $E_{0}\left(e^{-\lambda \tau_{b}}\right)=0$ for $\lambda>0$ and thus $P_{0}\left(\tau_{b}<\infty\right)=0$. If $\int^{b-} m(x) d x<\infty$, then, by Lemma $5.7, \varphi(b, \lambda)$ and $\psi(b, \lambda)$ are finite. Letting $\lambda \rightarrow 0$ in (6.4), we have

$$
\begin{aligned}
P_{0}\left(\tau_{b}<\infty\right) & =\left|l_{1}\right| /\left(\left|l_{1}\right|+b\right) & & \text { in case } l_{1}=l_{1}(m)>-\infty, \\
& =1 & & \text { in case } l_{1}=-\infty .
\end{aligned}
$$

Note that $l_{1}<0$ since $m(0-)=0$ for $m \in \mathscr{M}$. Thus $P_{0}\left(\tau_{b}<\infty\right)>0$. This completes the proof.

Since the conditional hitting time distribution $\mu(d t)=\mu_{0 b}(d t)$ is defined for $0 \in E_{m}$ and $b \in \tilde{E}_{m}$ satisfying $P_{0}\left\{\tau_{b}<\infty\right\}>0, \int^{b-} m(x) d x<\infty$ always holds. Let

$$
h_{2}(\lambda)=\psi(b, \lambda) / \varphi(b, \lambda)
$$

and

$$
h(\lambda)=\left(h_{1}(\lambda)^{-1}+h_{2}(\lambda)^{-1}\right)^{-1}
$$

Then we have

$$
E_{0}\left(e^{-\lambda \tau_{0}}\right)=h(\lambda) / \psi(b, \lambda) .
$$

Proof of the necessity part of Theorem 1. Let $m \in \mathscr{M}$. Let $0 \in E_{m}$, $b \in \tilde{E}_{m}, b>0$ and let $\mu=\mu_{0 b}$ be the conditional hitting time distribution of $b$ starting at 0 of the g.d.p. corresponding to $\mathscr{M}$. Then, by the above argument, we can represent $\mathscr{L} \mu(\lambda)$ using $\psi$ and $h$ defined by (6.2) and (6.6), respectively, as

$$
\mathscr{L} \mu(\lambda)=h(\lambda) / \psi(b, \lambda)
$$

Let

$$
\mathscr{L} \mu_{1}(\lambda)=b / \psi(b, \lambda)
$$

and

$$
\begin{aligned}
\mathscr{L} \mu_{2}(\lambda) & =h(\lambda)\left(\left|l_{1}\right|+b\right)\left(b\left|l_{1}\right|\right)^{-1} & & \text { if } l_{1}>-\infty \\
& =h(\lambda) / b & & \text { if } l_{1}=-\infty
\end{aligned}
$$

Since $0<b<\infty$ and $\int^{b-} m(x) d x<\infty$, by Lemma 5.7 and (3.1), we see that $\mu_{1} \in C E_{+0}$. Noting that $\mathscr{L} \mu_{2}(0)=1$, we have that $\mu_{2} \in M E_{+}$by Lemma 3.1 and Remark 3.1. Let $\left\{a_{i}\right\}$ be the parameter sequence of $\mu_{1}$. We see 
that the support of the spectral measure of $1 /\left(\lambda h_{2}(\lambda)\right) \in \mathscr{H}_{0}$ coincides with the set $\left\{a_{i}\right\} \cup\{0\}$. It is clear by the definition of $h(\lambda)$ that the spectral measure of $1 /(\lambda h(\lambda))$ has a point mass on each $a_{i}$. Since $0 \in E_{m}, h_{1}(\infty-)$ or $h_{2}(\infty-)=0$ holds by Remark 5.2. This is equivalent to that $h(\infty-)=0$. This means that $\mu_{2}(\{0\})=0$. This proves the necessity of the condition.

Proof of the sufficiency part of Theorem 1. Let $\mu$ satisfy the condition of Theorem 1 and let $\mu=\mu * \mu_{2}$ be the decomposition of $\mu$ given in Theorem 1. Let $\left\{a_{i}\right\}_{i<0}$ be the parameter sequence of $\mathscr{L} \mu_{1}(\lambda)$. That is, $\left\{a_{i}\right\}=\phi$ or $\left\{a_{i}\right\}$ is an at most countable increasing sequence of positive numbers satisfying $\sum a_{i}^{-1}<\infty$ such that

$$
\begin{aligned}
\mathscr{L} \mu_{1}(\lambda) & =1 \quad \text { in the case }\left\{a_{i}\right\}=\phi \text { and } \\
& =\prod a_{i}\left(\lambda+a_{i}\right)^{-1} \quad \text { in the case }\left\{a_{i}\right\} \neq \phi .
\end{aligned}
$$

By the assumption of Theorem 1 , there are $c \geqq 0$ and a nonnegative measure $\tilde{\sigma}$ satisfying $\int_{[0, \infty)} \frac{1}{1+\xi} \tilde{\sigma}(d \xi)<\infty$ such that

$$
\left(\lambda \mathscr{L} \mu_{2}(\lambda)\right)^{-1}=c+\int_{[0, \infty)} \frac{1}{\lambda+\xi} \tilde{\sigma}(d \xi)
$$

and $\tilde{\sigma}$ has a point mass on each $a_{i}$ in the case $\left\{a_{i}\right\} \neq \phi$. Note that $\tilde{\sigma}(\{0\})=1$ since $\mathscr{L} \mu_{2}(0)=1$. Since $\mu_{2}(\{0\})=0, \mathscr{L} \mu_{2}(\infty-)=0$ and thus $c>0$ or $\tilde{\sigma}([0, \infty))=\infty$. Decompose $\tilde{\sigma}$ as $\tilde{\sigma}=\tilde{\sigma}_{1}+\tilde{\sigma}_{2}$ so that supp $\tilde{\sigma}_{2}=$ $\left\{a_{i}\right\} \cup\{0\}$ and $\tilde{\sigma}_{2}(\{0\})=1$. Note that $\tilde{\sigma}_{1}(\{0\})=0$. By Lemma 3.1 and Lemma 3.2 , there are measures $\sigma_{1}$ and $\sigma_{2}$ on $[0, \infty)$ such that

$$
\left[\int_{[0, \infty)} \frac{\lambda}{\lambda+\xi} \tilde{\sigma}_{1}(d \xi)\right]^{-1}=d+\int_{[0, \infty)} \frac{1}{\lambda+\xi} \sigma_{1}(d \xi)
$$

and

$$
\left[c \lambda+\int_{[0, \infty)} \frac{\lambda}{\lambda+\xi} \tilde{\sigma}_{2}(d \xi)\right]^{-1}=e+\int_{[0, \infty)} \frac{1}{\lambda+\xi} \sigma_{2}(d \xi)
$$

where $d=\left[\tilde{\sigma}_{1}[(0, \infty))\right]^{-1}$. Denote the functions in (6.8) and (6.9) by $h_{1}(\lambda)$ and $h_{2}(\lambda)$, respectively. We have

$$
\mathscr{L} \mu_{2}(\lambda)=\left(h_{1}(\lambda)^{-1}+h_{2}(\lambda)^{-1}\right)^{-1} .
$$

Note that either $e=\left[\tilde{\sigma}_{2}[(0, \infty))\right]^{-1}$ or $e=0$ according as $c=0$ or $c>0$. We show that either $d=0$ or $e=0$. In the case $c=0$, since $\tilde{\sigma}([0, \infty))$ $=\infty$ and since $\tilde{\sigma}=\tilde{\sigma}_{1}+\tilde{\sigma}_{2}$, either $\tilde{\sigma}_{1}([0, \infty))$ or $\tilde{\sigma}_{2}([0, \infty))=\infty$. This yields 
that $d$ or $e=0$. Note that $h_{1}(0)=\tilde{\sigma}_{1}(\{0\})^{-1}=\infty, h_{2}(0)=\tilde{\sigma}_{2}(\{0\})^{-1}=1$ and $h_{2}(\lambda)$ is meromorphic and its zero points $\left\{-a_{i}\right\}$ satisfy $\sum a_{i}^{-1}<\infty$. Thus, $h_{2}(\lambda)$ is written as

$$
h_{2}(\lambda)=\prod a_{i}^{-1}\left(\lambda+a_{i}\right) / \prod b_{i}^{-1}\left(\lambda+b_{i}\right)
$$

with $0 \leqq b_{1}<a_{1}<b_{2}<a_{2}<\cdots$. Here $\left\{b_{i}\right\}$ may be empty. See [16]. By the surjectivity of Krein's correspondence, there are $m_{1}$ and $m_{2} \in \mathscr{M}_{+}$corresponding to $h_{1}$ and $h_{2}$, respectively. Since $h_{2}(0)=1, l_{2}=l\left(m_{2}\right)=1$. The function $m_{2}$ satisfies either 1 or 2 of Lemma 5.6. If $m_{2}$ satisfies 2 , then $1=l_{2} \in \tilde{E}_{m_{2}}$. If $m_{2}$ satisfies 1 , then we can extend $m_{2}$ so that $1 \in E_{m_{2}}$ and $1<l_{2}\left(m_{2}\right)<\infty$ without changing $h_{2}(\lambda)$. We use the same symbol $m_{2}$ to represent the extended one. Since we have chosen $\tilde{\sigma}_{1}$ so that $\inf \{x \geqq 0$; $\left.\tilde{m}_{1}(x)>0\right\}=0$ for a function $\tilde{m}_{1} \in \mathscr{M}_{+}$corresponding to $\tilde{\sigma}_{1}$, and since $m_{1}$ is the inverse $\tilde{m}_{1}^{-1}$ of $\tilde{m}_{1}, m_{1}(0)=0$. Let

$$
\begin{aligned}
m(x) & =-m_{1}((-x)-) & & \text { for } x<0 \text { and } \\
& =m_{2}(x) & & \text { for } x>0 .
\end{aligned}
$$

Then, $m \in \mathscr{M}$. Let $\varphi$ and $\psi$ be the continuous solutions of (6.1) and (6.2), respectively, with the above $m$. Then $h_{1}(\lambda)$ and $h_{2}(\lambda)$ are represented by $\varphi$ and $\psi$ as

$$
h_{1}(\lambda)=-\lim _{x \rightarrow l_{1}} \psi(x, \lambda) / \varphi(x, \lambda)
$$

and

$$
h_{2}(\lambda)=\lim _{x \rightarrow 1} \psi(x, \lambda) / \varphi(x, \lambda)=\psi(1, \lambda) / \varphi(1, \lambda) .
$$

Since $\psi(1,0)=1$ and since the set of zero points of $\psi(1, \lambda)$ coincides with $\left\{-a_{i}\right\}$

$$
\mathscr{L} \mu_{1}(\lambda)=1 / \psi(1, \lambda)
$$

by Lemma 5.7 and (6.7). Thus, we have, by (6.10) and (6.11),

$$
\mathscr{L} \mu(\lambda)=\mathscr{L} \mu_{1}(\lambda) \mathscr{L} \mu_{2}(\lambda)=\left\{\psi(1, \lambda)\left(h_{1}(\lambda)^{-1}+h_{2}(\lambda)^{-1}\right)\right\}^{-1} .
$$

Since $d=0$ or $e=0,0 \in E_{m}$. Therefore, $\mu$ is the hitting time distribution of 1 starting at 0 of the generalized diffusion process corresponding to $m$. The proof is complete.

Remark 6.1. Let $\mu$ be a gamma distribution with Laplace transform $\mathscr{L} \mu(\lambda)=(a /(a+\lambda))^{\alpha}, 0<\alpha<1, a>0$. Then the following hold: 
(i) The spectral measure $\sigma$ of $\mathscr{L} \mu(\lambda)$ is absolutely continuous and its density $s(x)$ is given by

$$
\begin{aligned}
s(x) & =0 & & \text { for } 0<x<a, \\
& =\frac{\sin \alpha \pi}{\pi}\left(\frac{a}{x-a}\right)^{\alpha} & & \text { for } x>a .
\end{aligned}
$$

(ii) The spectral measure of $(\lambda \mathscr{L} \mu(\lambda))^{-1}$ has a point mass 1 at the origin and absolutely continuous on $(0, \infty)$.

Proof of Remark 6.1. (i) Since

$$
\int_{a}^{\infty}\left(\frac{a}{u-a}\right)^{\alpha} e^{-x u} d u=\Gamma(1-\alpha) a^{\alpha} x^{\alpha-1} e^{-a x}
$$

and since $\Gamma(\alpha) \Gamma(1-\alpha)=\pi / \sin \alpha \pi$, we have

$$
\int_{0}^{\infty} s(u) e^{-x u} d u=\Gamma(\alpha)^{-1} a^{\alpha} x^{\alpha-1} e^{-a x} .
$$

This means that $s(u)$ is the density of $\sigma$.

(ii) Let $\tilde{\sigma}$ be the spectral measure of $h(\lambda)=(\lambda \mathscr{L} \mu(\lambda))^{-1}$. By Fatou's lemma [3], $\tilde{\sigma}$ is obtained by

$$
\tilde{\sigma}[u, v]=-\lim _{y \downarrow 0} \frac{1}{\pi} \int_{-v}^{-u} \operatorname{Im} h(x+i y) d x
$$

at continuity points $u$ and $v$ of $\tilde{\sigma}$. Let us calculate $\operatorname{Im} h(\lambda)$. Note that $h(\lambda)$ is real on $\mathbf{R}_{+}$. We have

$$
\operatorname{Im} h(\lambda)=\left(\frac{|a+\lambda|}{a}\right)^{\alpha}|\lambda|^{-1} \sin \left(\alpha \operatorname{Arccot} \frac{a+x}{y}-\operatorname{Arccot} \frac{x}{y}\right) .
$$

Since

$$
|\operatorname{Im} h(\lambda)| \leqq\left\{(x+a)^{2}\right\}^{\alpha / 2} a^{-\alpha}|x|^{-1}, \quad \lambda=x+i y,
$$

we have, by Lebesgue's dominated convergence theorem,

$$
\tilde{\sigma}[u, v]=\frac{\sin (1-\alpha) \pi}{\pi} \int_{(-v) \wedge(-a)}^{(-u) \wedge(-a)}|x|^{-1}\left|1+\frac{x}{a}\right|^{\alpha} d x
$$

for $0<u<v$. Here, $a \wedge b$ denotes $\min \{a, b\}$. Thus $\tilde{\sigma}$ is absolutely continuous on $(0, \infty)$ and its density is given by

$$
\begin{array}{rlrl}
s(x) & =0 & \text { for } 0<x<a, \\
& =\frac{\sin (1-\alpha) \pi}{\pi x}\left(\frac{x-a}{a}\right)^{\alpha} & & \text { for } x>a .
\end{array}
$$


The point mass $\tilde{\sigma}(\{0\})$ is obtained by

$$
\tilde{\sigma}(\{0\})=\lim _{\lambda \downarrow 0} \lambda h(\lambda)=\lim _{\lambda \downarrow 0}\left(\frac{a}{a+\lambda}\right)^{-\alpha}=1 .
$$

Proof of Corollary 2. Since every gamma distribution $\mu$ with exponent $\alpha \leqq 1$ belongs to $M E_{+}$and does not have a point mass at 0 , (i) is immediate from Corollary 1. Let us prove (ii). Let $\mu$ be a gamma distribution with Laplace transform $\mathscr{L} \mu(\lambda)=(a /(a+\lambda))^{\alpha}, \alpha>1, a>0$. Keeping (3.2) and (3.3) in mind, we see that in the case $\alpha>2$, it is impossible to decompose $\mathscr{L} \mu(\lambda)$ as $\mathscr{L} \mu_{1}(\lambda) \mathscr{L} \mu_{2}(\lambda)$ with $\mu_{1} \in C E_{+0}$ and $\mu_{2} \in M E_{+}$and in the case $1<\alpha \leqq 2$ only way is as follows:

$$
\mathscr{L} \mu(\lambda)=\left(\frac{a}{a+\lambda}\right)\left(\frac{a}{a+\lambda}\right)^{\alpha-1}
$$

If $1<\alpha<2$, then, by Remark 6.1, the spectral measure of $\left(\lambda(a /(a+\lambda))^{\alpha-1}\right)^{-1}$ is absolutely countinuous on $(0, \infty)$. If $\alpha=2$, then the spectral measure is concentrated at 0 . So in both cases, the spectral measure does not have a point mass at $a$. We conclude that $\mu \notin H_{g d}$.

LEMMA 6.1. (i) $H_{g d} \supset \mathrm{CME}_{+00}^{a}$.

(ii) Let $\mu \in H_{g d}$ and let $\mu_{1}$ and $\mu_{2}$ be the $C E_{+0}$ part and the $M E_{+0}$ part of $\mu$, respectively, in the representation in Theorem 1 . If the spectral measure of $\mathscr{L} \mu_{2}(\lambda)$ is discrete, then $\mu \in C M E_{+\infty 0}^{d}$.

Proof. (i) Let $\mu \in C M E_{+\infty 0}^{a}$ and let $\left(\left\{a_{i}\right\},\left\{b_{i}\right\},\left\{c_{i}\right\}\right)$ be the minimal representation of $\mu$. For each $i$, choose $a_{i}^{\prime}$ so that $a_{i}<a_{i}^{\prime}<a_{i+1}$ and the interval $\left(a_{i}, a_{i}^{\prime}\right)$ contains no point of $\left\{b_{j}\right\} \cup\left\{c_{k}\right\}$. Then the Laplace transform $\mathscr{L} \mu(\lambda)$ of $\mu$ is represented as

$$
\mathscr{L} \mu(\lambda)=\prod \frac{a_{i}^{\prime}}{a_{i}^{\prime}+\lambda} \prod \frac{\left(c_{i}^{\prime}+\lambda\right) b_{i}^{\prime}}{\left(b_{i}^{\prime}+\lambda\right) c_{i}^{\prime}}
$$

where $\left\{b_{i}^{\prime}\right\}=\left\{b_{i}\right\} \cup\left\{a_{i}\right\}, \quad\left\{c_{i}^{\prime}\right\}=\left\{c_{i}\right\} \cup\left\{a_{i}^{\prime}\right\}$. By the choice of $\left\{a_{i}^{\prime}\right\}, \prod b_{i}^{\prime} / c_{i}^{\prime}$ diverges to 0 in the case $\sharp\left\{b_{i}\right\}=\infty$. This representation shows that $\mathscr{L} \mu(\lambda)$ $\in H_{g d}$. Also we have that the spectral measure of $\prod\left(\left(c_{i}^{\prime}+\lambda\right) b_{i}^{\prime}\right) /\left(\left(b_{i}^{\prime}+\lambda\right) c_{i}^{\prime}\right)$ is discrete. (ii) By representing $\mathscr{L} \mu(\lambda)$ in the reduced form, we immediately get the conclusion.

Proof of Theorem 2. (i) Let $m \in \mathscr{M}$ satisfying 1,2 or 3 . In order to prove the assertion, we may assume that $0 \in E_{m}, 0<b \in \tilde{E}_{m}$ and $\mu$ is the 
conditional hitting time distribution of $b$ starting at 0 . As in the proof of Theorem 1 , decompose $\mathscr{L} \mu(\lambda)$ as $\mathscr{L} \mu(\lambda)=\mathscr{L} \mu_{1}(\lambda) \mathscr{L} \mu_{2}(\lambda)$ where

$$
\mathscr{L} \mu_{1}(\lambda)=b / \psi(b, \lambda)
$$

and

$$
\begin{aligned}
\mathscr{L} \mu_{2}(\lambda) & =h(\lambda)\left(\left|l_{1}\right|+b\right)\left(b\left|l_{1}\right|\right)^{-1} & & \text { if } l_{1}>-\infty \text { and } \\
& =h(\lambda) / b & & \text { if } l_{1}=-\infty .
\end{aligned}
$$

Here $\psi$ and $h$ are defined by (6.2) and (6.6) from $m$. It is clear that the spectral measure of $\left(\lambda h_{2}(\lambda)\right)^{-1}$ is discrete by Lemma 5.6. By the assumption and Lemma 5.5, we have that the spectral measure of $\left(\lambda h_{1}(\lambda)\right)^{-1}$ is discrete. Therefore the spectral measure of $(\lambda h(\lambda))^{-1}$ is discrete. This means that the spectral measure of $h(\lambda)$ is discrete. By Lemma (6) (ii), we get the conclusion $\mu \in C M E_{+\infty 0}^{d}$. (ii) Let $\mu \in C M E_{+00}^{d}$. Then, as in the proof of Lemma 6.1 (i), we can decompose $\mathscr{L} \mu(\lambda)$ as $\mathscr{L} \mu(\lambda)=\mathscr{L} \mu_{1}(\lambda) \mathscr{L} \mu_{2}(\lambda)$ where

$$
\mathscr{L} \mu_{1}(\lambda)=\prod \frac{a_{i}}{a_{i}+\lambda}, \quad \mathscr{L} \mu_{2}(\lambda)=\prod \frac{\left(c_{i}+\lambda\right) b_{i}}{\left(b_{i}+\lambda\right) c_{i}}, \quad\left\{a_{i}\right\} \subset\left\{c_{i}\right\}
$$

and $\prod \frac{b_{i}}{c_{i}}$ diverges to 0 in the case $\sharp\left\{b_{i}\right\}=\infty$ and $\sharp\left\{c_{i}\right\}=\#\left\{b_{i}\right\}-1$ in the case $\#\left\{b_{i}\right\}<\infty$. According to the method used in proving Theorem 1 , we can construct $h_{1}(\lambda)$ and $h_{2}(\lambda)$ so that

$$
\mathscr{L} \mu_{2}(\lambda)=\left(h_{1}(\lambda)^{-1}+h_{2}(\lambda)^{-1}\right)^{-1} .
$$

Since the support of the sepctral measure of $\left(\lambda h_{1}(\lambda)\right)^{-1}$ is contained in $\left\{c_{i}\right\}$, the specral measure of $h_{1}(\lambda)$ is discrete. By Lemma 5.5, the measure $m_{1} \in \mathscr{M}_{+}$corresponding to $h_{1}$ satisfies one of the conditions (1)-(3). The proof is complete.

\section{§ 7. Proof of Theorems 3-6}

In the proofs of Theorems 3 and 4 we use the notations $\varphi, \psi, h, h_{1}$, $h_{2}$ defined in Section 6 .

Proof of Theorem 3. Since $0<b<\infty$ and $\int_{0}^{b-} m(x) d x<\infty$, the support of the spectral measure $\sigma_{2}$ of $h_{2}$ is discrete by Lemma 5.6. Let $a_{21}=\inf \operatorname{supp} \sigma_{2}$. Since $b<\infty, a_{21}>0$ by Lemma 5.3. The function $h_{2}$ is meromorphic in $\mathbf{C}$ and, its zero points are negative and interlace the points in $-\operatorname{supp} \sigma_{2}=\left\{-a ; a \in \operatorname{supp} \sigma_{2}\right\}$. Let $-b_{21}$ be the largest zero of $h_{2}(\lambda)$. Then $-b_{21}<-a_{21}<0$ and $h_{2}(\lambda)>0$ on $\left(-a_{21}, 0\right)$. Let $\sigma_{1}$ be the 
spectral measure of $h_{1}$. (i) Let $a_{11}=\inf \operatorname{supp} \sigma_{1}$. Since $l_{1}>-\infty$, by Lemma 5.2 , we have $a_{11} \geqq 1 / 4 B>0$. Hence, $h_{1}(\lambda)$ is continued analytically to the interval $\left(-a_{11}, 0\right)$ and strictly decreasing in the interval. Now we see that $h_{1}^{-1}$ and $h_{2}^{-1}$ are positive and analytic in the interval $\left(-\left(a_{11} \wedge a_{21}\right), 0\right)$. Hence, the infimum of the support of the spectral measure $\sigma$ of $h$ is not less than $a_{11} \wedge a_{21}$. Since the largest zero of $\psi(b, \lambda)$ is $-b_{21}, h(\lambda) / \psi(b, \lambda)$ is analytic in $\left(-\left(a_{11} \wedge a_{21}\right), 0\right)$. In the case $\psi_{r} \equiv$ const., we directly obtain that, for each $0<c<a_{11} \wedge a_{21}$,

$$
e^{c t} \mu([t, \infty)) \longrightarrow 0 \quad \text { as } t \rightarrow \infty .
$$

In the case $\psi \not \equiv$ const., applying Cauchy's theorem to the inversion formula for Laplace transforms ([15], we get the same asymptotics. On the other hand, by Lemma 5.2 , we have

$$
a_{11} \geqq 1 / 4 B \text { and } a_{21} \geqq 1 / 4 A .
$$

We get the conclusion.

(ii) By Lemma 5.3, $\sigma_{1}(\{0\})>0$ and $\inf \left[\left.\left(\operatorname{supp} \sigma_{1}\right)\right|_{(0, \infty)}\right] \geqq 1 / 4 C>0$. Set $a_{12}=\inf \left[\left.\left(\operatorname{supp} \sigma_{1}\right)\right|_{(0, \infty)}\right]$. Hence $h_{1}(\lambda)$ is continued analytically to $\left(-a_{12}, 0\right)$ and strictly decreasing in $\left(-a_{12}, 0\right)$. Since the origin is a pole of $h_{1}(\lambda)$, there is unique zero $-b_{11}$ of $h_{1}$ in $\left(-a_{12}, 0\right)$. $h_{1}$ maps $\left[-b_{11}, 0\right)$ onto $(-\infty, 0]$. Hence there is only one zero $-\alpha$ of $h_{1}+h_{2}$ in $\left(-\left(a_{21} \wedge b_{11}\right), 0\right)$. Although the origin is a pole of $h_{1}$, it is cancelled with the numerator of

$$
h(\lambda)=h_{1}(\lambda) h_{2}(\lambda) /\left(h_{1}(\lambda)+h_{2}(\lambda)\right)
$$

and thus, $-\alpha$ is unique pole of $h(\lambda)$ in $\left(-\left(a_{21} \wedge b_{11}\right), 0\right]$. The zeros of $\psi(b, \lambda)$ are less than $-\alpha$ since $h_{2} \in \mathscr{H}_{0}$. Hence $-\alpha$ is the largest pole of $\mathscr{L} \mu(\lambda)$. Note that $-b_{11}$ is the largest pole of $1 / \lambda h_{1}(\lambda)$. Set

$$
D=\sup _{0<x<m_{1}(\infty-)}\left(m_{1}(\infty)-x\right) m_{1}^{-1}(x),
$$

where $m_{1}(x)=m((-x)-)$ for $x>0 . \quad$ Then $C=D . \quad$ By Lemma 5.2, we have

Hence

$$
1 / 4 C \leqq b_{11} \leqq 1 / C
$$

$$
\alpha<(1 / C) \wedge(1 / A)
$$

In the case $\psi \not \equiv$ const., applying the residue theorem to the inversion formula for Laplace transforms, we see that there is $M>0$ such that

$$
\mu\left(([t, \infty)) \sim M e^{-\alpha t} \quad \text { as } t \rightarrow \infty .\right.
$$


In the case $\psi \equiv$ const., we directly get the same asymptotics. The proof is complete.

Proof of Theorem 4. By Lemma 5.8, we have

$$
h_{1}(\lambda) \sim D_{\alpha} \lambda^{-\alpha} L_{\alpha}\left(\lambda^{-1}\right) \quad \text { as } \lambda \rightarrow 0 .
$$

Note that

$$
h_{2}(0)-h_{2}(\lambda)=O(\lambda) \quad \text { as } \lambda \rightarrow 0
$$

since $h_{2}(\lambda)$ is analytic near $\lambda=0$. By (7.1) and (7.2), we have

$$
h(0)-h(\lambda) \sim b^{2} \lambda^{\alpha} /\left\{D_{\alpha} L_{\alpha}\left(\lambda^{-1}\right)\right\} \quad \text { as } \lambda \rightarrow 0 .
$$

This yields

$$
\mathscr{L} \mu(0)-\mathscr{L} \mu(\lambda)=h(0) / \psi(b, 0)-h(\lambda) / \psi(b, \lambda) \sim b \lambda^{\alpha} /\left\{D_{\alpha} L_{\alpha}\left(\lambda^{-1}\right)\right\} .
$$

By the Tauberian theorem, we have

$$
\begin{aligned}
\mu([t, \infty)) & \sim b t^{-\alpha} /\left\{\Gamma(1-\alpha) D_{\alpha} L_{\alpha}(t)\right\} \\
& =b\{\alpha(1-\alpha)\}^{\alpha} /\left\{\Gamma(1+\alpha) t^{\alpha} L_{\alpha}(t)\right\} \quad \text { as } t \rightarrow \infty .
\end{aligned}
$$

The proof is complete.

In order to prove Theorems 5 and 6 , we can not apply Lemma 5.8 directly. We prepare some lemmas.

Lemma 7.1. Let $g$ and $h$ be functions in $\mathscr{H}_{0}$ which are $n(\geqq 0)$ times differential in a right neighborhood of the origin such that $g(0) \neq 0$. Assume that there are a function $L$ slowly varying at 0 , real numbers $a$, $b$ and $0<\alpha<1$ such that

$$
g(x)=\sum_{k=0}^{n} \frac{g^{(k)}(0)}{k !} x^{k}+(a+o(1)) x^{n+\alpha} L(x)
$$

and

$$
h(x)=\sum_{k=0}^{n} \frac{h^{(k)}(0)}{k !} x^{k}+(b+o(1)) x^{n+a} L(x)
$$

as $x \downarrow 0$. Then $f(x)=h(x) / g(x)$ is $n$ times differentiable and

$$
f(x)=\sum_{k=0}^{n} \frac{f^{(k)}(0)}{k !} x^{k}+(M+o(1)) x^{n+\alpha} L(x)
$$

as $x \downarrow 0$ where $M=(b g(0)-a h(0)) / g(0)^{2}$. 
Proof. It is easy to get in the case $n=0$. Let $n \geqq 1$. Since $g, h \in \mathscr{H}_{0}$, the derivatives of $g(x)-\sum_{k=0}^{n} \frac{g^{(k)}(0)}{k !} x^{k}$ and $h(x)-\sum_{k=1}^{n} \frac{h^{(k)}(0)}{k !} x^{k}$ are monotone in $x>0$. Then formulas (7.3) and (7.4) imply that $g, h \in C^{n}[0, \infty)$ and

$$
\begin{aligned}
& g^{(n)}(x)-g^{(n)}(0) \sim c(n+\alpha, n) a x^{\alpha} L(x), \\
& h^{(n)}(x)-h^{(n)}(0) \sim c(n+\alpha, n) b x^{\alpha} L(x)
\end{aligned}
$$

as $x \downarrow 0$, respectively, where $c(n+\alpha, n)=(n+\alpha)(n-1+\alpha) \cdots(1+\alpha)$. Since $g(0) \neq 0$, it is obvious that $f$ is $n$ times continuously differentiable on $[0, \infty)$. Differentiating $h(x)=f(x) g(x) n$ times, we have by Leibniz's formula that

$$
f^{(n)}(x) g(x)=h^{(n)}(x)-\sum_{k=0}^{n-1}{ }_{n} \mathrm{C}_{k} f^{(k)}(x) g^{(n-k)}(x)
$$

We have

$$
f^{(n)}(x)-f^{(n)}(0)=p(x)+q(x)
$$

where

$$
p(x)=g(x)^{-1}\left[\left\{h^{(n)}(x)-h^{(n)}(0)\right\}-\sum_{k=0}^{n-1}{ }_{n} \mathrm{C}_{k}\left\{f^{(k)}(x) g^{(n-k)}(x)-f^{(k)}(0) g^{(n-k)}(0)\right\}\right]
$$

and

$$
q(x)=f^{(n)}(0)\{g(0)-g(x)\} / g(x) .
$$

By (7.6) we have

$$
\begin{aligned}
p(x) & =g(0)^{-1}\left[h^{(n)}(x)-h^{(n)}(0)-f(0)\left\{g^{(n)}(x)-g^{(n)}(0)\right\}\right]+O(x) \\
& =c(n+\alpha, n) g(0)^{-1}\{b-a h(0) / g(0)+o(1)\} x^{\alpha} L(x)+O(x)
\end{aligned}
$$

and

$$
q(x)=O(x)
$$

as $x \downarrow 0$. Hence we have that

$$
f^{(n)}(x)-f^{(n)}(0) \sim c(n+\alpha, n) M x^{a} L(x) \quad \text { as } x \downarrow 0
$$

with $M$ in the lemma. This shows that $f^{(n)}(x)$ varies regularly at the origin. Integrating (7.7) $n$ times, we get (7.5). The proof is complete.

Lemma 7.2. For $h \in \mathscr{H}_{0}$ with $0<h(0)<\infty$, define $h^{*} \in \mathscr{H}_{0}$ by $h^{*}(\lambda)=$ $1 /\{\lambda h(\lambda)\}-1 /\{\lambda h(0)\}$. Let $0<\alpha<1$ and let $L$ be a function slowly varying at 0 . 
(i) If

$$
h^{\sharp}(\lambda)=\lambda^{\alpha-1} L(\lambda) \quad \text { as } \lambda \downarrow 0,
$$

then

$$
h(\lambda)=h(0)-\left(h(0)^{2}+o(1)\right) \lambda^{\alpha} L(\lambda) \quad \text { as } \lambda \downarrow 0 .
$$

(ii) If $h^{\sharp}$ is $n(n \geqq 0)$ times differentiable in a right neighborhood of 0 and satisfies

$$
h^{\sharp}(\lambda)=\sum_{k=0}^{n} \frac{\left(h^{\sharp}\right)^{(k)}(0)}{k !} \lambda^{k}+\lambda^{n+\alpha} L(\lambda)
$$

as $\lambda \downarrow 0$, then $h(\lambda)$ is $n+1$ times differentiable in a neighborhood of 0 and satisfies

$$
h(\lambda)=\sum_{k=0}^{n+1} \frac{h^{(k)}(0)}{k !} \lambda^{k}-\left(h(0)^{2}+o(1)\right) \lambda^{n+1+\alpha} L(\lambda) \quad \text { as } \lambda \downarrow 0 .
$$

Proof. (i) By the definition of $h^{\sharp}(\lambda)$, we have

$$
\frac{1}{h(\lambda)}-\frac{1}{h(0)}=\lambda h^{\sharp}(\lambda)=\lambda^{\alpha} L(\lambda) \quad \text { as } \lambda \downarrow 0 .
$$

Thus, we have the conclusion.

(ii) Set $g(\lambda)=\lambda h^{\sharp}(\lambda)$. Then, since

$$
g^{(n)}(\lambda)=\lambda\left(h^{\sharp}\right)^{(n)}(\lambda)+n\left(h^{\sharp}\right)^{(n-1)}(\lambda),
$$

we have

$$
\left\{g^{(n)}(\lambda)-g^{(n)}(0)\right\} / \lambda \longrightarrow(n+1)\left(h^{\sharp}\right)^{(n)}(0) \quad \text { as } \lambda \downarrow 0 .
$$

Thus $g(\lambda)$ is $n+1$ times differentiable at $\lambda=0$. Set $\tilde{g}(\lambda)=g(\lambda)+h(0)^{-1}$. By (7.9), the definition of $g$ and the uniqueness of Taylor's formula, we have

$$
\tilde{\boldsymbol{g}}(\lambda)=\sum_{k=0}^{n+1} \frac{\tilde{\boldsymbol{g}}^{(k)}(0)}{k !} \lambda^{k}+\lambda^{n+1+\alpha} L(\lambda) \quad \text { as } \lambda \downarrow 0 .
$$

Applying Lemma 7.1 to $h(\lambda)=1 / \tilde{g}(\lambda)$, we have (7.10) by (7.11). The proof is complete.

Lemma 7.3. Let $m \in \mathscr{M}_{+}$satisfying $l(m)=\infty$ and $m(\infty-)<\infty$. Set $h^{*}(\lambda)=h(\lambda)-\sigma(\{0\}) / \lambda$ where $\sigma$ is the spectral measure of $h$. Assume that for $\alpha>0(\alpha \neq 1)$ 


$$
m(\infty-)-m(x) \sim x^{-\alpha /(1+\alpha)} L(x) \quad \text { as } x \rightarrow \infty
$$

with a function $L$ slowly varying at $\infty$. Then we have the following for $m^{*}=\Phi^{-1}\left(h^{*}\right)$ :

(i) In the case $0<\alpha<1$, it holds that $l^{*}=l\left(m^{*}\right)=\infty$ and

$$
m^{*}(x) \sim x^{(1-\alpha)-1-1} M_{\alpha}(x) \quad \text { as } x \rightarrow \infty,
$$

where $x^{(1+\alpha) /(1-\alpha)}\left\{M_{\alpha}(x)\right\}^{2}(x \rightarrow \infty)$ is an asymptotic inverse of $C t^{(1-\alpha) /(1+\alpha)}\{L(t)\}^{2}$. Here

$$
C=m(\infty-)^{-4 /(1+\alpha)}((1+\alpha) /(1-\alpha))^{2 \alpha /(1+\alpha)} .
$$

(ii) In the case $\alpha>1$, it holds that $l^{*}<\infty$ and

$$
m^{*}(x) \sim\left(l^{*}-x\right)^{-(\alpha-1)-1-1} M_{\alpha}\left(l^{*}-x\right) \quad \text { as } x \uparrow l^{*},
$$

where $x^{(1+\alpha) /(1-\alpha)}\left\{M_{\alpha}(x)\right\}^{2}(x \downarrow 0)$ is an asymptotic inverse of $C t^{(1-\alpha) /(1+\alpha)}\{L(t)\}^{2}$. Here $C$ is the constant defined by (7.12).

Proof. Note that $\sigma(\{0\})>0$ by Lemma 5.3. (i) Let $0<\alpha<1$. In this case, by Lemma 5.4 we have $l^{*}=x(\infty-)=\infty$ and

$$
x(t) \sim \frac{1+\alpha}{1-\alpha} m(\infty-)^{-2} t^{(1-\alpha) /(1+\alpha)}\{L(t)\}^{2} \quad \text { as } t \rightarrow \infty .
$$

Setting $C^{\prime}=C m(\infty-)^{2}(1-\alpha) /(1+\alpha)$, we have, by the definition of $M_{\alpha}$,

$$
t(x) \sim\left(C^{\prime} x\right)^{(1+\alpha) /(1-\alpha)}\left\{M_{\alpha}(x)\right\}^{2} \quad \text { as } x \rightarrow \infty .
$$

We have, by Lemma 5.4, that

$$
\begin{aligned}
m^{*}(x) & \sim m(\infty-)^{2} t^{\alpha /(1+\alpha)} / L(t) \\
& \sim m(\infty-)^{2}\left(C^{\prime} x\right)^{\alpha /(1-\alpha)}\left\{M_{\alpha}(x)\right\}^{2 \alpha /(1+\alpha)} / L(t(x))
\end{aligned}
$$

as $x \rightarrow \infty$. By the definition of $M_{\alpha}(x)$, we have

$$
L(t(x)) \sim C^{-1 / 2}\left\{M_{\alpha}(x)\right\}^{(\alpha-1) /(\alpha+1)}
$$

as $x \rightarrow \infty$. Hence, substitutig (7.14) into (7.13), we have

$$
m^{*}(x) \sim x^{\alpha /(1-\alpha)} M_{\alpha}(x) .
$$

(ii) Let $\alpha>1$. In this case, by Lemma 5.4 we have $l^{*}=x(\infty-)<\infty$ and

$$
l^{*}-x(t) \sim m(\infty-)^{-2}\left(\frac{1+\alpha}{1-\alpha} t\right)^{(1-\alpha) /(1+\alpha)}\{L(t)\}^{2} \quad \text { as } t \rightarrow \infty .
$$


We have, by the definition of $M_{\alpha}$,

$$
t(x) \sim\left(C^{\prime}\left(l^{*}-x\right)\right)^{(1+\alpha) /(1-\alpha)}\left\{M_{\alpha}\left(l^{*}-x\right)\right\}^{2} \quad \text { as } x \rightarrow l^{*} .
$$

As in the proof of (i), use Lemma 5.4 and the definition of $M_{\alpha}$. We get the conclusion of (ii). The proof is complete.

Lemma 7.4. Let $m \in \mathscr{M}_{+}$. Assume that $l=l(m)<\infty$ and $m(l-)=\infty$. If there are $\alpha>0(\alpha \neq 1)$ and a function $L(x)$ slowly varying at 0 such that

$$
m(x) \sim(l-x)^{\alpha-1-1} L(l-x) \quad \text { as } x \rightarrow l,
$$

then the following hold:

(i) If $0<\alpha<1$, then $l^{\sharp}=l\left(\left(m^{-1}\right)^{*}\right)=\infty$ and

$$
\left(m^{-1}\right)^{*}(x) \sim x^{(1-\alpha)-1-1} M(x) \quad \text { as } x \rightarrow \infty
$$

with a function $M$ slowly varying at $\infty$.

(ii) If $\alpha>1$, then $l^{\sharp}<\infty$ and

$$
\left(m^{-1}\right) *(x) \sim\left(l^{\sharp}-x\right)^{-1 /(\alpha-1)-1} M\left(l^{\sharp}-x\right) \quad \text { as } x \uparrow l^{\sharp}
$$

with a function $M$ slowly varying at 0 . Here * stands for the operation defined in Lemma 7.3.

Proof. We have

$$
m^{-1}(\infty-)-m^{-1}(x) \sim x^{-\alpha /(1+\alpha)} M_{\alpha}(x) \quad \text { as } x \rightarrow \infty,
$$

where $x^{-\alpha /(1+\alpha)} M_{a}(x)$ is an asymptotic inverse of $t^{-\alpha-1-1} L(t)(t \rightarrow 0)$. The conclusions in (i) and (ii) are direct from Lemma 7.3 (i) and (ii), respectively. The proof is complete.

LEMmA 7.5. Let $m \in \mathscr{M}_{+}$satisfying $l=l(m)<\infty$ and $m(l-)=\infty$. Let $\alpha$ be a non-integral positive number and $n$ be an integer satisfying $n<\alpha<n+1$. If there is a function $L$ slowly varying at 0 such that

$$
m(x) \sim(l-x)^{-\alpha^{-1-1}} L(l-x) \quad \text { as } x \rightarrow l
$$

then $h(\lambda)=\Phi(m)(\lambda)$ is $n$ times differentiable in a right neighborhood of 0 and satisfies

$$
h(\lambda)=\sum_{k=0}^{n} \frac{h^{(k)}(0)}{k !} \lambda^{k}+(-1)^{n+1} \lambda^{\alpha} M(\lambda) \quad \text { as } \lambda \downarrow 0
$$

with a function $M$ slowly varying at 0 . 
Proof. Let $\tilde{m}$ be a function defined by the $n+1$ times iteration of $\#: m \rightarrow\left(m^{-1}\right)^{*}$ to $m$. Then, by Lemma 7.4 , we have

$$
\tilde{m}(x) \sim x^{(n+1-\alpha)-1-1} K(x) \quad \text { as } x \rightarrow \infty
$$

where $K$ is a function slowly varying at $\infty$. By Lemma 5.8, there is a function $N$ slowly varying at 0 such that

$$
\tilde{h}(\lambda)=\Phi(\tilde{m})(\lambda) \sim \lambda^{-(n+1-\alpha)} N(\lambda) \quad \text { as } \lambda \rightarrow 0 .
$$

By Lemmas 5.1, 3 and 4, we see that the function $\tilde{h}$ is obtained from $h$ iterating the operation \# defined in Lemma $7.2 n+1$ times. Taking Lemma 7.2 into consideration, we have the conclusion by (7.8) and (7.10). The proof is complete.

Lemma 7.6. Let $\mu \in \mathscr{P}\left(R_{+}\right)$and let $h(\lambda)=\mathscr{L} \mu(\lambda)$ be the Laplace transform of $\mu$. If $n$-th right derivative $h^{(n)}(0)$ exists, then the following hold:

(i) $\mu$ has $k$-th moments for $k \leqq n$.

$$
F_{k}(t)=\int_{t}^{\infty} \int_{t_{k-1}}^{\infty} \cdots \int_{t_{1}}^{\infty} \mu\left(d t_{0}\right) d t_{1} \cdots d t_{k-1},
$$

$1 \leqq k \leqq n+1$, is well defined and has $(n-k)$-th moment $\int_{0}^{\infty} t^{n-k} F_{k}(t) d t$.

$$
\int_{0}^{\infty} e^{-\lambda t} F_{k}(t) d t=(-1)^{k} \lambda^{-k}\left\{h(\lambda)-\sum_{j=0}^{k-1} \frac{h^{(j)}(0)}{j !} \lambda^{j}\right\} \text {. }
$$

Proof. (i) Clearly, $\mu$ has $k$-th moment $(k \leqq n)$ and

$$
\int_{0}^{\infty} t^{k} \mu(d t)=(-1)^{k} h^{(k)}(0) \text {. }
$$

(ii) Note that $F_{k}(t)=\int_{t}^{\infty} F_{k-1}(t) d t$ for $2 \leqq k \leqq n$. In order to prove that $F_{k}(t), 1 \leqq k \leqq n$, is well defined and has $(n-k)$-th moment, it is enough to show that $\int_{0}^{\infty} t^{k} \mu(d t)<\infty$ if and only if $\int_{0}^{\infty} t^{k-1} \mu[t, \infty) d t<\infty$. Moreover, we see that

$$
\int_{0}^{\infty} t^{k-1} \mu[t, \infty) d t=k^{-1} \int_{0}^{\infty} t^{k} \mu(d t) .
$$

Let us show the above fact. Let $M>0$. Integrating by parts we have

$$
\int_{0}^{M} t^{k-1} \mu[t, \infty) d t=k^{-1}\left[M^{k} \mu[M, \infty)+\int_{[0, M)} t^{k} \mu(d t)\right] .
$$

Thus, if $\int_{0}^{\infty} t^{k-1} \mu[t, \infty) d t<\infty$, then $\int_{0}^{\infty} t^{k} \mu(d t)<\infty$. Conversely, if $\int_{0}^{\infty} t^{k} \mu(d t)$ 
$<\infty$, then since $t^{k} \mu[t, \infty) \leqq \int_{[t, \infty)} s^{k} \mu(d s)$, we have $t^{k} \mu[t, \infty) \rightarrow 0$ as $t \rightarrow \infty$. Hence $\int_{0}^{\infty} t^{k-1} \mu[t, \infty) d t$ is finite and equals $k^{-1} \int_{0}^{\infty} t^{k} \mu(d t)$. Thus (7.16) holds. By (7.16), we have

$$
F_{k+1}(0)=\frac{1}{k !} \int_{0}^{\infty} t^{k} \mu(d t)
$$

(iii) We represent $\mathscr{L} F_{k}(\lambda)$ by $h$ and its derivatives by induction in $k$. For $k=1$, integrating by parts, we have

$$
\int_{0}^{\infty} e^{-\lambda t} F_{1}(t) d t=\lambda^{-1}\left[\mu[0, \infty)-\int_{0}^{\infty} e^{-\lambda t} \mu(d t)\right]=\lambda^{-1}\{h(0)-h(\lambda)\} .
$$

Assume that (iii) is true for $k \leqq l$. Integrating by parts and using (7.17), we have

$$
\begin{aligned}
\int_{0}^{\infty} e^{-\lambda t} F_{l+1}(t) d t & =\lambda^{-1}\left[F_{l+1}(0)-\int_{0}^{\infty} e^{-\lambda t} F_{l}(t) d t\right] \\
& =\lambda^{-1}\left[(l !)^{-1} \int_{0}^{\infty} t^{l} \mu(d t)-\int_{0}^{\infty} e^{-\lambda s} F_{l}(t) d t\right] .
\end{aligned}
$$

We have, by (7.15), (7.18) and the assumption of induction,

$$
\int_{0}^{\infty} e^{-\lambda t} F_{l+1}(t) d t=(-1)^{l+1} \lambda^{-l-1}\left\{h(\lambda)-\sum_{j=0}^{l} \frac{h^{(1)}(0)}{j !} \lambda^{j}\right\} .
$$

This completes the proof.

LEMma 7.7. Let $\mu \in \mathscr{P}\left(\mathbf{R}_{+}\right)$. If the Laplace transform $\mathscr{L} \mu(\lambda)$ of $\mu$ is $n$ times differentiable in a right neighborhood of 0 and saitsfies

$$
\mathscr{L} \mu(\lambda)=\sum_{k=0}^{n} \frac{(\mathscr{L} \mu)^{(k)}(0)}{k !} \lambda^{k}+(-1)^{n+1} \lambda^{\alpha} K(\lambda)
$$

as $\lambda \downarrow 0$ with $n<\alpha<n+1$ and a function $K$ slowly varying at 0 , then

$$
\mu[t, \infty) \sim A t^{-\alpha} K(1 / t) \quad \text { as } t \rightarrow \infty
$$

where $A=\{\Gamma(\alpha+1) \sin \pi(\alpha-n)\} / \pi$.

Proof. By Lemma 7.6,

$$
F_{n+1}(t)=\int_{t}^{\infty} \int_{t_{n}}^{\infty} \cdots \int_{t_{1}}^{\infty} \mu\left(d t_{0}\right) d t_{1} \cdots d t_{n}
$$

is well defined and its Laplace transform satisfies 


$$
\int_{0}^{\infty} e^{-\lambda t} F_{n+1}(t) d t=\lambda^{\alpha-n-1} K(\lambda) \quad \text { as } \quad \lambda \downarrow 0 .
$$

By the Tauberian theorem,

$$
F_{n+1}(t) \sim \frac{1}{\Gamma(n+1-\alpha)} t^{-(\alpha-n)} K(1 / t) \quad \text { as } \quad t \rightarrow \infty .
$$

Note that $F_{k}(t)(1 \leqq k \leqq n+1)$ are monotone functions. Differentiating $F_{n+1}(t) n$ times, we have

$$
\mu[t, \infty)=F_{1}(t) \sim A t^{-\alpha} K(1 / t) \quad \text { as } \quad t \rightarrow \infty
$$

The proof is complete.

Proof of Theorem 5. Let $n$ be a nonnegative integer satisfying $n<\alpha$ $<n+1$. Let $m_{1}=m((-x)-)$ for $x>0$. Let $\psi, h_{1}, h_{2}$ and $h$ be functions defined by (6.2), (6.3), (6.5) and (6.6), respectively. By Lemma 7.5, $h_{1}=\Phi\left(m_{1}\right)$ is $n$ times differentiable in a right neighborhood of 0 and satisfies

$$
h_{1}(\lambda)=\sum_{k=0}^{n} \frac{h_{1}^{(k)}(0)}{k !} \lambda^{k}-(-1)^{n+1} \lambda^{\alpha} M(\lambda) \quad \text { as } \quad \lambda \downarrow 0
$$

with a function $M$ slowly varying at 0 . Write the function $h$ as $h(\lambda)=$ $h_{1}(\lambda) / g(\lambda)$ where $g(\lambda)=1+h_{1}(\lambda) / h_{2}(\lambda)$. Noting that $h_{2}$ is analytic near the origin, we have that $g(\lambda)$ is $n$ times differentiable in a right neighborhood of 0 and satisfies

$$
g(\lambda)=\sum_{k=0}^{n} \frac{g^{(k)}(0)}{k !} \lambda^{k}+(-1)^{n+1}\left(\frac{1}{h_{2}(0)}+o(1)\right) \lambda^{a} M(\lambda)
$$

as $\lambda \downarrow 0$. Since $g(0)>0$, we can apply Lemma 7.1 to $h$. We have that $h$ is $n$ times differentiable in a right neighborhood of 0 and satisfies

$$
h(\lambda)=\sum_{k=0}^{n} \frac{h^{(k)}(0)}{k !} \lambda^{k}+(-1)^{n+1}(A+o(1)) \lambda^{\alpha} M(\lambda)
$$

as $\lambda \downarrow 0$ where $A=\left[h_{2}(0) /\left(h_{1}(0)+h_{2}(0)\right)\right]^{2}>0$. Since $\psi(b, \lambda)$ is an entire function of $\lambda$ and $\psi(b, 0)=b$,

$$
\mathscr{L} \mu(\lambda)=\sum_{k=0}^{n} \frac{(\mathscr{L} \mu)^{(k)}(0)}{k !} \lambda^{k}+(-1)^{n+1}(A / b+o(1)) \lambda^{\alpha} M(\lambda)
$$

as $\lambda \downarrow 0$. By Lemma 7.7, we get the conclusion. The proof is complete.

Proof of Theorem 6. Let $m_{1}(x)=m((-x)-)$ for $x>0$. Let $\psi, h_{1}, h_{2}$ 
and $h$ be functions defined by (6.2), (6.3), (6.5) and (6.6), respectively. Then $h_{1}=\Phi\left(m_{1}\right)$. Define $h_{1}^{*}$ as in Lemma 7.3 for $h_{1}$. Set $f(\lambda)=\lambda h_{1}(\lambda)$. Let $n$ be an integer such that $n<\alpha<n+1$. Let us show that the function $f$ is $n$ times differentiable in a right neighborhood of 0 and satisfies

$$
f(\lambda)=\sum_{k=0}^{n} \frac{f^{(k)}(0)}{k !} \lambda^{k}+(-1)^{n}(1+o(1)) \lambda^{\alpha} N(\lambda)
$$

as $\lambda \downarrow 0$. Let $0<\alpha<1$. Then, by Lemma 7.3(i),

$$
m_{1}^{*}(x)=\Phi^{-1}\left(h_{1}^{*}\right)(x) \sim x^{(1-\alpha)-1-1} M(x) \quad \text { as } \quad x \rightarrow \infty
$$

with a function $M$ slowly varying at $\infty$. By Lemma 5.8,

$$
h_{1}^{*}(\lambda) \sim \lambda^{\alpha-1} N(\lambda) \quad \text { as } \quad \lambda \downarrow 0
$$

with a function $N$ slowly varying at 0 . This implies (7.19). Now let $\alpha>1$. Then by Lemma 7.3 (ii), $l^{*}=l\left(m_{1}^{*}\right)<\infty$ and

$$
m_{1}^{*}(x) \sim\left(l^{*}-x\right)^{-(\alpha-1)-1-1} M\left(l^{*}-x\right) \quad \text { as } \quad x \rightarrow l^{*}
$$

with a function $M$ slowly varying at 0 . By Lemma 7.5, we have

$$
h_{1}^{*}(\lambda)=\sum_{k=0}^{n-1} \frac{h_{1}^{*(k)}(0)}{k !} \lambda^{k}+(-1)^{n}(1+o(1)) \lambda^{\alpha-1} N(\lambda)
$$

sa $\lambda \downarrow 0$ with a function $N$ slowly varying at 0 . We have (7.19) in a similar way for $\tilde{g}(\lambda)$ in the proof of Lemma 7.2. Note that, by Lemma 5.3,

$$
f(0)=\lim _{\lambda \downarrow 0} \lambda h_{1}(\lambda)=1 / m_{1}(\infty-)>0 .
$$

The function $h(\lambda)$ is written as follows:

$$
h(\lambda)=f(\lambda) h_{2}(\lambda) /\left\{f(\lambda)+\lambda h_{2}(\lambda)\right\}=h_{2}(0)+\lambda q(\lambda) / p(\lambda)
$$

where

$$
p(\lambda)=f(\lambda)+\lambda h_{2}(\lambda)
$$

and

$$
q(\lambda)=f(\lambda)\left\{h_{2}(\lambda)-h_{2}(0)\right\} / \lambda-h_{2}(0) h_{2}(\lambda) .
$$

By (7.19), the functions, $p$ and $q$ are $n$ times differentiable in a right neighborhood of 0 and satisfy

$$
p(\lambda)=\sum_{k=0}^{n} \frac{p^{(k)}(0)}{k !} \lambda^{k}+(-1)^{n}(1+o(1)) \lambda^{\alpha} N(\lambda)
$$


as $\lambda \downarrow 0$ and

$$
q(\lambda)=\sum_{k=0}^{n} \frac{q^{(k)}(0)}{k !} \lambda^{k}+(-1)^{n}\left(h_{2}^{\prime}(0)+o(1)\right) \lambda^{a} N(\lambda)
$$

as $\lambda \downarrow 0$. Let us apply Lemma 7.1 to $r(\lambda)=q(\lambda) / p(\lambda)$. Note that $p(0)=f(0)$ and $q(0)=f(0) h_{2}^{\prime}(0)-h_{2}(0)^{2}$. Thus the constant $M$ appearing in Lemma 7.1 for $r(\lambda)$ is given by $M=(-1)^{n} M^{\prime}$ where $M^{\prime}=\left\{h_{2}(0) / f(0)\right\}^{2}>0$. Hence we have, by Lemma 7.1 .

$$
h(\lambda)=h(0)+\lambda\left\{\sum_{k=0}^{n} \frac{r^{(k)}(0)}{k !} \lambda^{k}+(-1)^{n}\left(M^{\prime}+o(1)\right) \lambda^{\alpha} N(\lambda)\right\}
$$

as $\lambda \downarrow 0$. Since $\psi(b, \lambda)$ is an entire function of $\lambda$,

$$
\mathscr{L} \mu(\lambda)=\sum_{k=0}^{n+1} \frac{(\mathscr{L} \mu)^{(k)}(0)}{k !} \lambda^{k}+(-1)^{n+2}\left(M^{\prime} / b+o(1)\right) \lambda^{\alpha+1} N(\lambda)
$$

as $\lambda \downarrow 0$. By Lemma 7.7, we have

$$
\mu[t, \infty) \sim t^{-\alpha-1} L(t) \quad \text { as } t \rightarrow \infty
$$

where $L$ is a function slowly varying at infinity. This completes the proof.

\section{REFERENCES}

[1] Bondesson, L., Classes of infinitely divisible distributions and densities, Z. Wahrsch. Verw. Gebiete, 57 (1981), 39-71.

[2] Donoghue, W. F., Jr., Monotone matrix functions and analytic continuation, Springer, Berlin Heidelberg New York, 1974.

[ 3 ] Dym, H. and McKean, H. P., Gaussian processes, function theory, and the inverse spectral problem, Academic press, New York San Francisco London, 1976.

[4] Has'minskii, R. Z., Stochastic stability of differential equations, Sijthoff \& Noordhoff, Alphen aan den Rijn Rockville, 1980.

[ 5 ] Ito, K. and McKean H. P., Jr., Dffusion processes and their sample paths, second printing, Springer, Berlin Heidelberg New York, 1974.

[6 ] Kac, I. S. and Krein, M. G., On the spectral functions of the string, A. M. S. Translations Series 2, Vol. 103 (1974), 19-102, (original in Russian, "Mir", Moscow (1968), 648-737).

[ 7 ] - Criteria for the discreteness of the spectrum of a singular string, Izv. Vyss. Ucebn. Zaved. Mat., 2 (1958), 136-153, (in Russian).

[8] Kasahara, Y., Spectral theory of generalized second order differential operators and its applications to Markov processes, Japan J. Math., 1 (1975), 67-84.

[ 9 ] Keilson, J., On the unimodality of passage time densities in birth-death processes, Statist. Neerlandica, 35 (1981), 49-55.

[10] Kent, J. T., Eigenvalue expansions for diffusion hitting times, Z. Wahrsch. Verw. Gebiete, 52 (1980), 309-319.

[11] Krein, M. G., On some classes of the effective determination of the densities of a non-homogeneous string from its spectral function, Dokl. Acad. Nauk SSSR 93 
(1953), 617-620 (in Russian).

[12] Kotani, S. and Watanabe, S., Krein's spectral theory of strings and generalized diffusion processes, Functional Analysis in Markov Processes (M. Fukushima, ed.), Lecture Notes in Math., 923 (1982), 235-259, Springer, Berlin Heidelberg New York.

[13] Seneta, E., Regularly varying functions, Lecture notes in Math., 58 (1976), Springer, Berlin Heidelberg New York.

[14] Stone, C., Limit theorems for random walks, birth and death processes, and diffusion processes, Illinois J. Math., 7 (1963), 638-660.

[15] Widder, D. V., The Laplace Transform, Princeton Univ. Press, Princeton, 1972.

[16] Yamazato, M., Characterization of the class of upward first passage time distributions of birth and death processes and related results, J. Math. Soc. Japan, 40 (1988), 477-499.

Department of Mathematics

Nagoya Institute of Technology

Gokiso, Showa-ku

Nagoya, 466

Japan 